\title{
Almost symplectic structures on the linear frame bundle from linear connection
}

\begin{abstract}
We describe all $\mathcal{M} f_{m}$-natural operators $S: Q \rightsquigarrow S y m p P^{1}$ transforming classical linear connections $\nabla$ on $m$-dimensional manifolds $M$ into almost symplectic structures $S(\nabla)$ on the linear frame bundle $P^{1} M$ over $M$.
\end{abstract}

Let $V$ be a real vector space of even dimension. A bilinear form $\varpi: V \times$ $V \rightarrow \mathbb{R}$ is called a symplectic form if it is antisymmetric and nondegenerate, i.e. it satisfies

$\varpi(v, v)=0$ for all $v \in V$ and if $\varpi(v, u)=0$ for all $v \in V$, then $u=0$.

A vector space $V$ is a symplectic vector space if it is equipped with a symplectic form, [1].

Let $\mathcal{M} f_{m}$ denote the category of $m$-dimensional manifolds and their embeddings and $\mathcal{F} \mathcal{M}$ denote the category of fibred manifolds and fibred maps between them.

For any $m$-dimensional manifold $M$ we have the linear frame bundle $P^{1} M=\operatorname{inv} J_{0}^{1}\left(\mathbb{R}^{m}, M\right)$ of the manifold $M$. This is a principal bundle with corresponding Lie group $G L(m)=G_{m}^{1}=i n v J_{0}^{1}\left(\mathbb{R}^{m}, \mathbb{R}^{m}\right)_{0}$, which acts on $P^{1} M$ on the right via compositions of jets. Every map $\psi: M_{1} \rightarrow M_{2}$ from the category $\mathcal{M} f_{m}$ induces a map $P^{1} \psi: P^{1} M_{1} \rightarrow P^{1} M_{2}$ by $P^{1} \psi\left(j_{0}^{1} \varphi\right)=$ $j_{0}^{1}(\psi \circ \varphi)$, where $\varphi: \mathbb{R}^{m} \rightarrow M_{1}$ is a map from the category $\mathcal{M} f_{m}$. The correspondence $P^{1}: \mathcal{M} f_{m} \rightarrow \mathcal{F M}$ is a bundle functor in the sense of [3].

2000 Mathematics Subject Classification. 58A20.

Key words and phrases. Classical linear connection, almost symplectic structure, linear frame bundle, natural operator. 
For any $2 n$-dimensional manifold $N$ we have an almost symplectic structures bundle $\operatorname{Symp}(N)=\bigcup_{y \in N} \operatorname{Sym} p\left(T_{y} N\right)$ over the manifold $N$, where $\widetilde{\operatorname{Sym} p}\left(T_{y} N\right)$ denotes the set of symplectic forms $\varpi: T_{y} N \times T_{y} N \rightarrow \mathbb{R}$ on the tangent space $T_{y} N$. The bundle $\operatorname{Symp}(N)$ is a subbundle (but not vector subbundle) of a vector bundle $T^{*} N \otimes T^{*} N$ of tensors of type $(0,2)$ over $N$. Sections $\Omega: N \rightarrow \operatorname{Symp}(N)$ are called almost symplectic structures on the manifold $N$. Every embedding $\psi: N_{1} \rightarrow N_{2}$ induces a fibred map $\operatorname{Symp}(\psi): \operatorname{Symp}\left(N_{1}\right) \rightarrow \operatorname{Symp}\left(N_{2}\right)$ being restriction of $T^{*} \psi \otimes T^{*} \psi: T^{*} N_{1} \otimes T^{*} N_{1} \rightarrow T^{*} N_{2} \otimes T^{*} N_{2}$ to $\operatorname{Symp}(N)$. The correspondence Symp: $\mathcal{M} f_{2 n} \rightarrow \mathcal{F M}$ is a bundle functor in the sense of [3].

Let $M$ be an $m$-dimensional manifold. We have the classical linear connection bundle $Q M:=\left(i d_{T^{*} M} \otimes \pi^{1}\right)^{-1}\left(i d_{T M}\right) \subset T^{*} M \otimes J^{1} T M$ of the manifold $M$, where $\pi^{1}: J^{1} T M \rightarrow T M$ is the projection of the first jet prolongation $J^{1} T M=\left\{j_{x}^{1} X: X \in \mathfrak{X}(M), x \in M\right\}$ of the tangent bundle $T M$ of the manifold $M$. Sections $\tilde{\nabla}: M \rightarrow Q M$ correspond bijectively to classical linear connections on $M$. Every embedding $f: M_{1} \rightarrow M_{2}$ induces a fibred map $Q f: Q M_{1} \rightarrow Q M_{2}$ covering $f$. The correspondence $Q: \mathcal{M} f_{m} \rightarrow \mathcal{F M}$ is a bundle functor in the sense of [3].

Let $\left\{A_{i}^{j *}\right\}, i, j=1, \ldots, m$ be the standard basis in $\mathfrak{g l}(m)=\mathcal{L} i e(G L(m))$.

For a principal fibre bundle $P^{1} M$ the action of group $G L(m)$ on $P^{1} M$ induces a homomorphism $\sigma$ of Lie algebra $\mathfrak{g l}(m)$ of group $G L(m)$ into Lie algebra $\mathfrak{X}\left(P^{1} M\right)$ of vector fields on $P^{1} M$. For every $A \in \mathfrak{g l}(m)$, a vector field $A^{*}=\sigma(A)$ is called the fundamental vector field corresponding to $A$. Since the action of group $G L(m)$ on $P^{1} M$ sends each fibre into itself, therefore $A_{u}^{*}$ is tangent to the fibre at each $u \in P^{1} M,[2]$.

Let $\nabla$ be a classical linear connection on $m$-dimensional manifold $M$. For every $\xi \in \mathbb{R}^{m}$ we define the standard horizontal vector field $B(\xi)$ on $P^{1} M$ as follows. For each $u \in P^{1} M, u: \mathbb{R}^{m} \rightarrow T_{\pi(u)} M$, a vector $(B(\xi))_{u}$ is the unique horizontal vector at $u$ such that $T \pi\left((B(\xi))_{u}\right)=u(\xi)$, where $\pi: P^{1} M \rightarrow M,[2]$.

The canonical form $\theta$ of bundle $P^{1} M$ is $\mathbb{R}^{m}$-valued 1-form on $P^{1} M$ defined by

$$
\theta(X)=u^{-1}(T \pi(X)) \quad \text { for } X \in T_{u}\left(P^{1} M\right),
$$

where $\pi: P^{1} M \rightarrow M$ and $u: \mathbb{R}^{m} \rightarrow T_{\pi(u)}(M),[2]$.

For a given connection $\nabla$ on $P^{1} M$ we define a 1-form $\omega$ on $P^{1} M$ with values in Lie algebra $\mathfrak{g l}(m)$ of group $G L(m)$ as follows. For each $X \in$ $T_{u}\left(P^{1} M\right)$ we define $\omega(X)$ to be the unique $A \in \mathfrak{g l}(m)$ such that $\left(A^{*}\right)_{u}$ is equal to the vertical component of vector $X$. The form $\omega$ is called the connection form of the given connection $\nabla,[2]$.

Let $B_{1}, \ldots, B_{m}$ be the standard horizontal vector fields corresponding to basic vectors $e_{1}, \ldots, e_{m}$ of space $\mathbb{R}^{m}$ and let $\left\{A_{i}^{j *}\right\}$ be fundamental vector fields corresponding to basic vectors $\left\{A_{i}^{j}\right\}$ of Lie algebra $\mathfrak{g l}(m)$. It is easy 
to verify that $\left\{B_{l}, A_{i}^{j *}\right\}$ and $\left\{\theta^{i}, \omega_{j}^{i}\right\}$ are dual to each other, i.e. they satisfy

$$
\begin{aligned}
& \theta^{k}\left(B_{l}\right)=\delta_{l}^{k}, \quad \theta^{k}\left(A_{i}^{j *}\right)=0, \\
& \omega_{r}^{k}\left(B_{l}\right)=0, \quad \omega_{r}^{k}\left(A_{i}^{j *}\right)=\delta_{i}^{k} \delta_{r}^{j},
\end{aligned}
$$

where $\theta^{i}$ are components of the canonical 1-form and $\omega_{j}^{i}$ are components of the connection form.

Proposition 1 ([2]). The $m^{2}+m$ vector fields $\left\{B_{k}, A_{i}^{j^{*}} ; i, j, k=1, \ldots, m\right\}$ define an absolute parallelism in the bundle $P^{1} M$.

The following definition of a natural operator is particular case of an idea of natural operator which was considered in [3].

Definition 1. An $\mathcal{M} f_{m}$-natural operator $S: Q \rightsquigarrow S y m p P^{1}$ is a family of $\mathcal{M} f_{m}$-invariant regular operators $S=\left(S_{M}\right)$

$$
S_{M}: \underline{Q}(M) \rightarrow \underline{\operatorname{Symp}}\left(P^{1} M\right)
$$

for any manifold $M$ from the category $\mathcal{M} f_{m}$, where $Q(M)$ is the set of all linear connections on the manifold $M$ (sections of $Q(M) \rightarrow M$ ) and $\operatorname{Symp}\left(P^{1} M\right)$ is the set of all almost symplectic structures on $P^{1} M$ (sections of $\left.\operatorname{Symp}\left(P^{1} M\right) \rightarrow P^{1} M\right)$. The invariance means that if $\nabla_{1} \in Q\left(M_{1}\right)$ and $\nabla_{2} \in Q\left(M_{2}\right)$ are $\psi$-related by $\psi: M_{1} \rightarrow M_{2}$, that is $Q(\psi) \circ \nabla_{1}=\nabla_{2} \circ \psi$, then $\overline{S\left(\nabla_{1}\right)}$ and $S\left(\nabla_{2}\right)$ are $P^{1} \psi$-related, that is $\operatorname{Symp}\left(P^{1} \psi\right) \circ S\left(\nabla_{1}\right)=$ $S\left(\nabla_{2}\right) \circ P^{1} \psi$. The regularity means that smoothly parametrized families of classical linear connections are transformed by $S$ on smoothly parametrized families of almost symplectic structures.

In the present note we will classify all natural operators $S$ and obtained result will be modification of result in [4].

Remark 1. In [4] there were described geometric constructions on higher order frame bundles $P^{r} M$. In the present paper we describe only case of linear frame bundle $P^{1} M$. The generalization of this problem for $P^{r} M$ is not possible, because dimension of $P^{r} M$ for $r>1$ does not have to be even.

For given connection $\nabla \in \underline{Q}(M)$ with respect to the global basis of vector fields $\left\{B_{k}, A_{i}^{j *}\right\}$ on $P^{1} M$ we have a canonical (in $\nabla$ ) fibred diffeomorphism

$$
K_{\nabla}: P^{1} M \times \widetilde{\operatorname{Sym} p}\left(\mathbb{R}^{m^{2}+m}\right) \rightarrow \operatorname{Symp}\left(P^{1} M\right)
$$

covering $i d_{P^{1} M}$ defined by the condition that the matrix of map $K_{\nabla}(u(x), \varpi)$ in the basis $\left\{B_{k}(\nabla)(u(x)), A_{i}^{j *}(u(x))\right\}$ is the same as the one of the symplectic form $\varpi$ in the canonical basis of space $\mathbb{R}^{m^{2}+m}$. 
Let $Z^{s}=J_{0}^{s}\left(Q\left(\mathbb{R}^{m}\right)\right), s=0,1, \ldots, \infty$ be the set of $s$-jets $j_{0}^{s} \nabla$ of all classical linear connections $\nabla$ on $\mathbb{R}^{m}$ satisfying

$$
\sum_{j, k=1}^{m} \nabla_{j k}^{i}(x) x^{j} x^{k}=0 \text { for } i=1, \ldots, m,
$$

it means that the usual coordinates $x^{1}, \ldots, x^{m}$ on $\mathbb{R}^{m}$ are $\nabla$-normal with center $0 \in \mathbb{R}^{m}$.

Example 1. General construction: Let $\mu: Z^{\infty} \rightarrow \widetilde{\operatorname{Sym} p}\left(\mathbb{R}^{m^{2}+m}\right)$ be a map satisfying the following local finite determination property.

For any $\rho \in Z^{\infty}$ we can find an open neighborhood $U \subset Z^{\infty}$ of jet $\rho$, a natural number $s$ and a smooth map $f: \pi_{s}(U) \rightarrow \widetilde{S y m p}\left(\mathbb{R}^{m^{2}+m}\right)$ such that $\mu=f \circ \pi_{s}$ on $U$, where $\pi_{s}: Z^{\infty} \rightarrow Z^{s}$ is the jet projection. (A simple example of such $\mu$ is $\mu=f \circ \pi_{s}$ for smooth $f: Z^{s} \rightarrow \widetilde{\operatorname{Sym} p}\left(\mathbb{R}^{m^{2}+m}\right)$ and for finite number $s$.)

Given a classical linear connection $\nabla$ on an $m$-dimensional manifold $M$ we define an almost symplectic structure $S^{\langle\mu\rangle}(\nabla)$ on $P^{1} M$ as follows. Let $u(x) \in\left(P^{1} M\right)_{x}, x \in M$. Choose a $\nabla$-normal coordinate system $\psi$ on $M$ with center $x$ such that $P^{1} \psi(u(x))=l^{0}=j_{0}^{1}\left(i d_{\mathbb{R}^{m}}\right)$. Such a coordinate system $\psi$ exists. Then $\operatorname{germ}_{x}(\psi)$ is uniquely determined. We put

$$
S^{\langle\mu\rangle}(\nabla)_{u(x)}=\operatorname{Symp}\left(P^{1}\left(\psi^{-1}\right)\right)\left(K_{\psi_{*} \nabla}\left(l^{0}, \mu\left(j_{0}^{\infty}\left(\psi_{*} \nabla\right)\right)\right)\right) .
$$

Since $\operatorname{germ}_{x}(\psi)$ is uniquely determined, then above definition is correct. The family $S^{\langle\mu\rangle}: Q \rightsquigarrow S y m p P^{1}$ is an $\mathcal{M} f_{m}$-natural operator.

Theorem 1. Any $\mathcal{M} f_{m}$-natural operator $S: Q \rightsquigarrow S y m p P^{1}$ is of the form $S^{<\mu>}$ for some uniquely determined (by $S$ ) function $\mu: Z^{\infty} \rightarrow \widetilde{S y m p}\left(\mathbb{R}^{m+m^{2}}\right)$ satisfying local finite determination property.

Proof. Let $S: Q \rightsquigarrow S y m p P^{1}$ be an $\mathcal{M} f_{m}$-natural operator. Define $\mu: Z^{\infty} \rightarrow$ $\widetilde{\operatorname{yym} p}\left(\mathbb{R}^{m+m^{2}}\right)$ by

$$
\left(l^{0}, \mu\left(j_{0}^{\infty} \nabla\right)\right)=K_{\nabla}^{-1}\left(S(\nabla)\left(l^{0}\right)\right) .
$$

Then by non-linear Peetre theorem, [3], $\mu$ satisfies local finite determination property. Then by definitions of $\mu$ and $S^{<\mu>}$ we have that $S(\nabla)\left(l^{0}\right)=$ $S^{<\mu>}(\nabla)\left(l^{0}\right)$ for any classical linear connection $\nabla$ on $\mathbb{R}^{m}$ such that the identity map $i d_{\mathbb{R}^{m}}$ is a $\nabla$-normal coordinate system with center $0 \in \mathbb{R}^{m}$. Then by the invariance of $S$ and $S^{<\mu>}$ with respect to normal coordinates we deduce that $S=S^{<\mu>}$.

Remark 2. Symplectic geometry methods are key ingredients in the study of dynamical systems, mathematical physics, analytical mechanics, differential geometry, [1], [5]. 


\title{
REFERENCES
}

[1] Berndt, R., An Introduction to Symplectic Geometry, Graduate Studies in Mathematics, Vol 26, American Mathematical Society, Providence, Rhode Island, 2001.

[2] Kobayashi, S., Nomizu, K., Foundations of Differential Geometry, Vol I, Interscience Publisher, New York-London, 1963.

[3] Kolář, I., Michor, P. W. and Slovák, J., Natural Operations in Differential Geometry, Springer-Verlag, Berlin, 1993.

[4] Kurek, J., Mikulski, W. M., Riemannian structures on higher order frame bundles from classical linear connections, Differential Geometry, Proceedings of the VIII International Colloquium Santiago de Compostela, World Scientific 2009, 296-300.

[5] León, M. de, Rodrigues, P. R., Methods of Differential Geometry in Analytical Mechanics, North-Holland Math. Stud. 158, Amsterdam, 1989.

\author{
Anna Bednarska \\ Institute of Mathematics \\ Maria Curie-Sklodowska University \\ pl. M. Curie-Sklodowskiej 1 \\ 20-031 Lublin \\ Poland \\ e-mail: bednarska@hektor.umcs.lublin.pl
}

Received June 22, 2009 